\title{
Simulation Studies of Some Voronoi Point Processes*
}

\author{
K.A. Borovkov ${ }^{\dagger}$ and D.A. Odell ${ }^{\ddagger}$ \\ December 7, 2017
}

\begin{abstract}
We introduce a new class of dynamic point process models with simple and intuitive dynamics that are based on the Voronoi tessellations generated by the processes. Under broad conditions, these processes prove to be ergodic and produce, on stabilisation, a wide range of clustering patterns. In the paper, we present results of simulation studies of three statistical measures (Thiel's redundancy, van Lieshout and Baddeley's $J$-function and the empirical distribution of the Voronoi nearest neighbours' numbers) for inference on these models from the clustering behaviour in the stationary regime. In particular, we make comparisons with the area-interaction processes of Baddeley and van Lieshout.
\end{abstract}

\section{Introduction}

In many diverse fields such as biology, geography, business and telecommunications, spatial point configurations evolve according to criteria dependent on a zone of influence in some general sense. A natural way of formalising this concept is based on using Voronoi tessellations (see e.g. 9]).

Let $(M, d)$ be a metric space and $N>0$ the initial number of points in our process. The associated configuration space $\mathscr{X}$ consists of all $N$-point subsets of $M: \mathscr{X}:=\{\boldsymbol{x} \subset$ $M: \operatorname{card}(\boldsymbol{x})=N\}$ ('multiple points' will be a.s. impossible in our models). For an $\boldsymbol{x} \in \mathscr{X}$, the Voronoi cell of $x_{i}$ relative to $\boldsymbol{x}$ is defined as

$$
C_{x_{i}}^{x}:=\left\{y \in M: d\left(y, x_{i}\right)=\min _{j \leq N} d\left(y, x_{j}\right)\right\} .
$$

The set $\mathscr{T}_{\boldsymbol{x}}=\left\{C_{x_{i}}^{\boldsymbol{x}}: x_{i} \in \boldsymbol{x}\right\}$ is called the Voronoi tessellation generated by $\boldsymbol{x}$.

We define a Voronoi point process as a discrete-time Markov processes $\left\{\boldsymbol{x}_{n}\right\}_{n \geq 0}$ with values in $\mathscr{X}$ which evolves as follows: at each step, a point is chosen from the current configuration $\boldsymbol{x}_{n}$ according to a probability rule determined by $\mathscr{T}_{\boldsymbol{x}_{n}}$ and removed from the configuration, and at the same time a new point is added at a random location, according to a fixed probability measure $\mu$ on $M$. Our initial interest interest in such dynamics was prompted by its relevance to some of the real-life processes in the above-mentioned application areas. In addition, simulations showed that processes constructed according to this model display very interesting forms of clustering behaviour, and constructing

\footnotetext{
*This research was supported by the ARC Centre of Excellence for Mathematics and Statistics of Complex Systems.

${ }^{\dagger}$ University of Melbourne, email: k.borovkov@ms.unimelb.edu.au

${ }^{\ddagger}$ MASCOS, Melbourne, email: d.odell@ms.unimelb.edu.au
} 
models of point processes producing desired clustering patterns is of substantial interest both theoretically and practically. (see e.g. 3, 9]).

Despite the fact that it is notoriously difficult to obtain analytic results for processes of such a nature, we were able to prove ergodicity in a number of interesting cases. To formulate the results, we first need to further specify the 'culling rule' in the model.

We assume that a non-negative 'selection function' $S$ is given on the set of all possible Voronoi cells in $M$. Then, given that $\boldsymbol{x}=\left\{x_{1}, \ldots, x_{N}\right\}$ is the current configuration of the point process, in the next step a random point $x_{J} \in \boldsymbol{x}$ is chosen to be removed, with the distribution of the index $J$ given by

$$
\mathbf{P}(J=j \mid \boldsymbol{x}):=\frac{S\left(C_{x_{j}}^{\boldsymbol{x}}\right)}{\sum_{i=1}^{N} S\left(C_{x_{i}}^{\boldsymbol{x}}\right)}, \quad 1 \leq J \leq N .
$$

The function $S$ can be based on different properties of a Voronoi cell. In this note, we consider only two of them: the 'volume' of the cell and the number of its edges. More precisely, we introduce the following two classes of Voronoi point processes:

(A) The volume-based Voronoi point process, or $v$-process, on $M=\mathbb{S}^{1}$ or $M=[0,1]^{2}$. Let $\lambda$ be the 'volume measure' on $M$ (length on $\mathbb{S}^{1}$, area on $[0,1]^{2}$ ). We assume that the value of the selection function $S\left(C_{x_{j}}^{x}\right)$ is determined by the volume of the cell $C_{x_{j}}^{x}$ : for a function $S_{v}: \mathbb{R}^{+} \mapsto \mathbb{R}^{+}$, one puts $S\left(C_{x_{j}}^{x}\right):=S_{v}\left(\lambda\left(C_{x_{j}}^{x}\right)\right.$ ). If $S_{v}$ is increasing, then points with Voronoi cells of large volume are more likely to be culled, and so the selection favours points with small cells, i.e. points restricted by 'close neighbours'. A decreasing $S_{v}$ favours points with large cells. Functions of the form $S_{v}(u)=u^{\alpha}, \alpha \in \mathbb{R}$, produce scale-independent models (in this case the dynamics of the process will clearly be invariant under scale transformations of $M$ ).

(B) The neighbour-based Voronoi point process, or $n$-process, on $M=[0,1]^{2}$. The set $\boldsymbol{x}\left[x_{j}\right]$ of Voronoi-nearest neighbours of a point $x_{j} \in \boldsymbol{x}$ is defined as the collection of those generators $x_{k} \in \boldsymbol{x}$ whose Voronoi cells share an edge with $C_{x_{j}}^{\boldsymbol{x}}$ :

$$
\boldsymbol{x}\left[x_{j}\right]:=\left\{x_{k} \in \boldsymbol{x}: k \neq j, \operatorname{card}\left(C_{x_{k}}^{\boldsymbol{x}} \cap C_{x_{j}}^{\boldsymbol{x}}\right)>1\right\} .
$$

We assume that, for a function $S_{n}:\{1, \ldots, N\} \mapsto \mathbb{R}^{+}$, we have $S\left(C_{x_{j}}^{\boldsymbol{x}}\right)=S_{n}\left(\operatorname{card}\left(\boldsymbol{x}\left[x_{j}\right]\right)\right)$.

In both cases (A) and (B), we assume the placement probability $\mu=\lambda$.

Theorem 1. (i) The neighbour-based Voronoi point process with a positive selection function $S_{n}:\{1, \ldots, N\} \mapsto \mathbb{R}^{+}$is Harris ergodic.

(ii) The volume-based Voronoi point process with a selection function $S_{v}:(0, \lambda(M)] \mapsto$ $\mathbb{R}^{+}$, such that both $S_{v}$ and $1 / S_{v}$ are bounded on closed subsets, is Harris ergodic.

Recall that Harris ergodicity entails convergence to the stationary distribution in total variation (see e.g. p.560 in [8] or p.154 in [2]). For the proof of the theorem, see [5].

This result justifies the approach taken in the present note, which is devoted to simulation studies of point patterns emerging in stationary regime in the evolution of Voronoi point processes. More specifically, we are interested in the performance of three relatively simple statistics one could employ to characterise the resulting stationary distributions. These are Thiel's redundancy measure (which is essentially the relative entropy for the set of cell volumes) and, in the two-dimensional case, the distribution of the number of Voronoi nearest neighbours for a random cell and the $J$-function of Baddeley and van 
Lieshout (which provides a comparison of the environment of a 'random point' of the configuration with that of a 'random point' of the underlying space over a range of scales). We demonstrate that the $n$ - and $v$-processes produce (for different choices of parameters) quite different clustering patterns, and that the combination of the above three measures work reasonably well in distinguishing between different Voronoi processes, and also between Voronoi processes and the area-interaction processes of Baddeley and Van Lieshout 3.

\section{Simulation Studies}

\section{$2.1 \quad$ One-dimensional $v$-processes}

The simple model of scale-free process on $M=\mathbb{S}^{1}$ with $S_{v}(u)=u^{\alpha}$ demonstrates that very interesting dynamics and point patterns arise even in one-dimension. This includes a 'phase change' observed when varying the value of $\alpha$. Figure 1 depicts side-by-side realisations of three different $v$-processes of this type, each having the same number of points $N=128$, but different $\alpha$ values. The base of each rectangle represents the circle $\mathbb{S}^{1}$ opened out into a line segment by a cut.

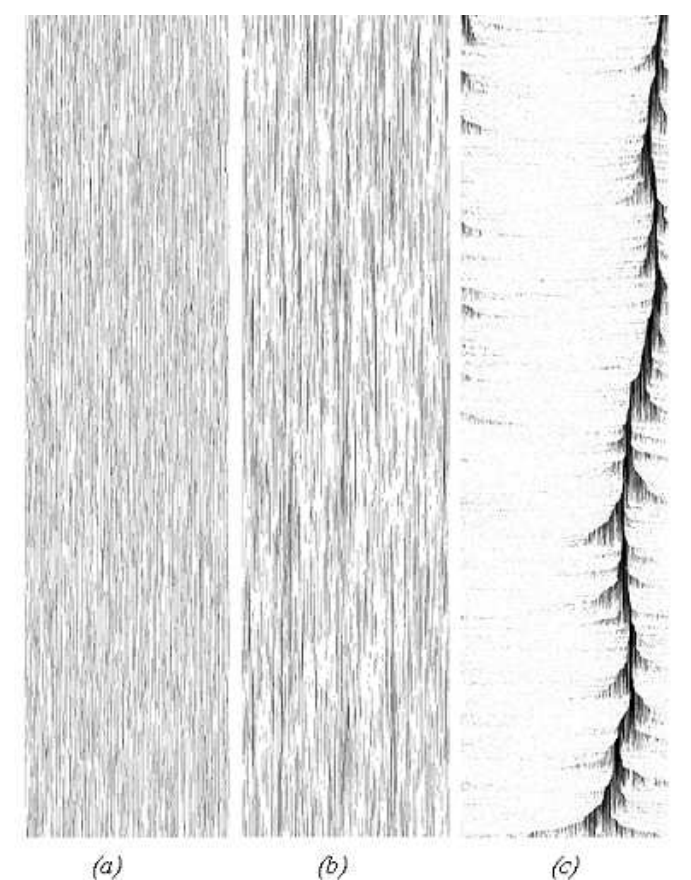

Figure 1: Evolution of $v$-processes on circle. Each of the three processes (with the selection functions $S_{v}(u)=u^{\alpha}$ with (a) $\alpha=-1.0$, (b) $\alpha=0.5$, (c) $\alpha=1.5$, resp.) was run with $N=128$ points for $T=4096$ steps (the vertical axis represents time).

We see a dramatic phase shift in behaviour between (b) and (c) that can be located more precisely at $\alpha=1$; its sharpness was found to increase with $N$. When $\alpha>1$, a cluster forms whose stability also increases with $N$. When $\alpha \leq 1$, we observe a degree of clustering in $M$ that varies with $\alpha$.

When $\alpha=0$, the points are uniformly distributed, so the associated Voronoi cell volumes have pretty nearly a Gamma distribution with shape parameter 2 (as cell-width is half the sum of two consecutive uniform spacings). For $\alpha \leq 1$, the histogram of 
cell volumes stabilises after a large number of steps to a result well-fitted by a Gamma distribution with a shape parameter varying inversely with $\alpha$. This suggests that the maximum likelihood estimator for the Gamma shape parameter for the cell-volume data could be a suitable statistic for inference on $\alpha$. Furthermore, under the assumption of Gamma distribution, the shape parameter can equivalently be estimated from the entropy - and the latter can also be used without the above assumption, namely in the form of Thiel's redundancy measure which is introduced as follows.

For a fixed configuration $\boldsymbol{x}$, let $p_{j}:=\lambda\left(C_{x_{j}}^{\boldsymbol{x}}\right) / \lambda(M), 1 \leq n \leq N$, be the probability that a random uniformly distributed point in $M$ lies in $C_{x_{i}}^{x}$. Then Thiel's redundancy measure $R^{*}(\boldsymbol{x})$ of $\boldsymbol{x}$ is defined [7, 9] as the entropy of the distribution $\left\{p_{j}\right\}_{j=1}^{N}$ relative to the uniform one on $\{1, \ldots, N\}$ :

$$
R^{*}(\boldsymbol{x}):=\ln N+\sum_{j=1}^{N} p_{j} \ln p_{j} .
$$

Simulations show that this statistic works quite well over a reasonably large range of $\alpha$ values: there is a very small variance in its values when $N \geq 10^{3}$, and a strong enough dependence on $\alpha$ to use $R^{*}$ for reliable estimation of the parameter. Figure 2 displays (connected) average values of 25 independent realizations for each of the values $\alpha=$ $-2+0.3 k, 0 \leq k \leq 10$, of $R^{*}\left(\boldsymbol{x}_{T}\right)$ after a large number of steps $T$ (cf. Fig. 3 ).

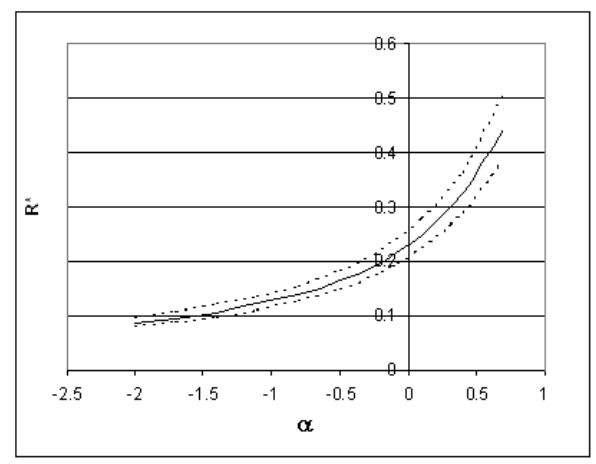

Figure 2: Empirical Thiel's redundancy measure for $v$-processes on $\mathbb{S}^{1}$ with $S_{v}(u)=u^{\alpha}, N=10^{3}$. The dotted lines show the $95 \%$ probability intervals.

The behaviour of the statistic $R^{*}\left(\boldsymbol{x}_{T}\right)$ as $T$ increases could also be used to estimate the rate of convergence to stationarity. Figure 3 (left) gives an indication of that rate for a range of $\alpha$ values. It shows that the rate is quite high for $\alpha<0.5$, whereas when $\alpha$ approaches one, it takes the process much longer to settle in a stable regime, and also that the oscillations of $\left\{R^{*}\left(\boldsymbol{x}_{T}\right)\right\}_{T \geq 0}$ are higher for those values of $\alpha$. At the threshold value $\alpha=1$, the sequence oscillates in quite diverse ranges of values demonstrating metastable beahviour, see Fig. 3 (right).

\subsection{Two dimensions: edge effects}

The problem of 'edge effects' (where the region boundary proximity can affect the shape/size of Voronoi cells) in simulations of Voronoi tessellations in (a rectangle) $M \subset \mathbb{R}^{2}$ is commonly dealt with by treating the underlying space as a torus or by considering only those parts of $M$ that lie within a window significantly distant from the edge $\partial M$ of $M$. This approaches is satisfactory for 'static' point processes [9]. In the case of the Voronoi 

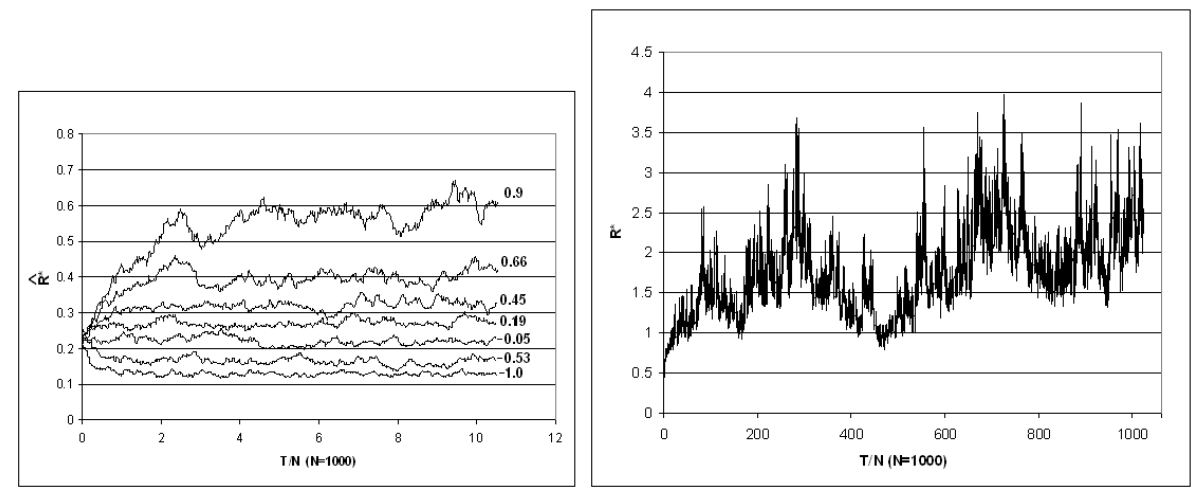

Figure 3: Evolution of $\left\{R^{*}\left(\boldsymbol{x}_{T}\right)\right\}_{T \geq 0}$ for $v$-processes on $\mathbb{S}^{1}: N=10^{3}, S_{v}(u)=u^{\alpha}$. Left: $\alpha=-1 ;-0.53$; $-0.05 ; 0.19 ; 0.45 ; 0.66 ; 0.9$, on the time interval $T \leq 10 N$. Right: $\alpha=1$, on the time interval $T \leq 10^{3} N$.

processes, edge effects may propagate in the course of the evolution, which presents an interesting problem by itself.

Let $\boldsymbol{x} \subset M=[0,1]^{2}$ be finite, $A \subset \mathbb{R}^{2}$. The Voronoi tessellation $\mathscr{T}_{\boldsymbol{x}}$ induces a nearestneighbour (NN) distance $d_{N N}^{x}\left(x_{j}, A\right)$ between $x_{j} \in \boldsymbol{x}$ and $A$, defined as the length of the shortest 'path' $x_{j}=x(1), \ldots, x(i) \in \boldsymbol{x}$ with the properties that the Voronoi cells of $x(m)$ and $x(m+1)$ have a common edge, $1 \leq m<i$, and $C_{x(i)}^{x} \cap A \neq \varnothing$. We can gauge the significance of edge effects by observing the changes in $R^{*}$ when we restrict attention to $x_{j} \in \boldsymbol{x}$ with $d_{N N}^{x}\left(x_{j}, \partial M\right) \geq m$ for a fixed $m>0$. Edge effects were assessed by a two-way ANOVA using NN distance as one factor, and the selection function as the other. In the case of the $v$-process with $S_{v}(u)=u^{\alpha}$, we found that edge effects were only significant in the layer of cells adjacent to the boundary, without any significant effect from $\alpha$. For the $n$-processes, significant edge effects were detected only for the 'anti-few' and 'anti-many' selection functions (see subsection 2.3 below), with no significant propagation beyond the NN-depth of two.

To minimise edge effects in our results, the statistics were generally computed from the set of cells with $d_{N N}^{x}\left(x_{j}, \partial M\right) \geq 3$.

\subsection{Two-dimensional $n$-processes}

In this family of Voronoi processes, the evolution is driven by one of the most natural local characteristics: the number of neighbours of the process points, i.e. the number of the edges of their Voronoi cells. For non-degenerate random configurations in $\mathbb{R}^{2}$, the vertices of the Voronoi tessellation a.s. terminate three edges, so by Euler's theorem the mean number of edges for a cell is six. There can be cells with any number of edges $m \geq 3$, although in our simulations cells with $m>13$ were rare, with relative frequency $<10^{-3}$.

We studied the $n$-processes on $M=[0,1]^{2}$ with the following selection functions: (i) $S_{n}(n)=n$ ('vanilla', in which cells with a large number of neighbours are more likely to be culled); (ii) $S_{n}(n)=n^{2}$ ('anti-many', which more severely penalises cells with large numbers of neighbours); (iii) $S_{n}(n)=(n-2)^{-2}$ ('anti-few', which does just the opposite); (iv) $S_{n}(n)=(0.1+|n-6|)^{-2}$ ('anti-6', which penalises cells with six or close to six neighbours); (v) $S_{n}(n)=|n-6|^{2}$ ('pro-6', which does the opposite); and 'sharp filters' focussing on cells with a given number neighbours: (vi) $S_{n}(5)=5000$ and $S(n)=1$ if $n \neq 5$ ('anti-5'); and (vii) $S_{n}(5)=1$ and $S_{n}(n)=5000$, if $n \neq 5$ ('pro-5'), and in addition the 'anti-' and 'pro-' selectors for four and seven. 
The statistics used in the study included:

(a) the empirical probability mass function (EPMF) for the number of Voronoi NN's,

(b) Thiel's redundancy measure, and also

(c) the $J$-function of van Lieshout and Baddeley [3].

The $J$-function $J(r)$ compares the 'environment' of a 'typical generator' of the process with that of a 'random point' in the underlying space, and is defined as the ratio of the probabilities that a disk of radius $r$ centred at the given point is empty of (other) generators of the process.

Formally, let $\boldsymbol{x}$ be a stationary isotropic point process in $\mathbb{R}^{2}$. Set

$B(r):=\left\{x \in \mathbb{R}^{2}:|x| \leq r\right\}, \quad F(r):=\mathbf{P}(\boldsymbol{x} \cap B(r) \neq \varnothing), \quad G(r):=\mathbf{P}^{! 0}(\boldsymbol{x} \cap B(r) \neq \varnothing)$,

where $\mathbf{P}^{! 0}$ is the reduced Palm distribution of $\boldsymbol{x}$ (the conditional distribution of $\boldsymbol{x} \backslash\{0\}$ given that there is a point at the origin). Then the $J$-function is defined as

$$
J(r):=\frac{1-G(r)}{1-F(r)} \quad \text { for all } r \geq 0 \text { such that } F(r)<1 .
$$

In the case of a Poisson point process of constant intensity, or complete spatial randomness (CSR), clearly $J(r) \equiv 1$, see e.g. [1]. If there is a tendency towards clustering obesrvable at scale $r$, then $J(r)<1$, while $J(r)>1$ if there is a tendency toward regular spacing and $r$ is of the order of the average distance between neighbours.

To estimate $J(r)$, one uses empirical distribution functions $\hat{F}(r)$ and $\hat{G}(r)$ :

$$
\hat{J}(r):=\frac{1-\hat{G}(r)}{1-\hat{F}(r)} .
$$

This is only reliably computable when $F(r) \leq 0.85$. Moreover, simulations showed that the values of $\hat{J}$ have relatively large variances when $N \asymp 10^{3}$, so we averaged the $\hat{J}$ values over 25-75 independent draws of the process, and performed a weighted cubic regression on the resulting points (with weights equal to the reciprocals of the standard deviations; higher order regressions add little to the goodness of fit).

The results of our study for a selection of the above-listed $n$-processes are summarised in Fig. 4 (statistics (c), (a)) and Table1 (statistic (b)).

\begin{tabular}{c|c|c|c}
\hline$n$-process & $\bar{R}^{*}$ & s.d. $\left(R^{*}\right)$ & s.e. $\left(\bar{R}^{*}\right)$ \\
\hline vanilla & 0.1450 & 0.0068 & 0.0014 \\
anti-many & 0.1548 & 0.0073 & 0.0015 \\
anti-few & 0.1074 & 0.0047 & 0.0009 \\
pro-6 & 0.1615 & 0.0083 & 0.0012 \\
anti-6 & 0.1524 & 0.0059 & 0.0008 \\
pro-5 & 0.1479 & 0.0067 & 0.0013 \\
anti-5 & 0.1255 & 0.0063 & 0.0013
\end{tabular}

Table 1: $R^{*}$ values for various $n$-processes, with standard deviations and standard errors. The values $\bar{R}^{*}$ are averages of 25 independent draws of $R^{*}(N=2000$, after $T=12 N$ steps $)$. For CSR, $R^{*}=0.135$.

The $J$-function curves provide the most nuanced 'picture' of the distribution patterns, but these curves were obtained by averaging over a number of observations of highly 


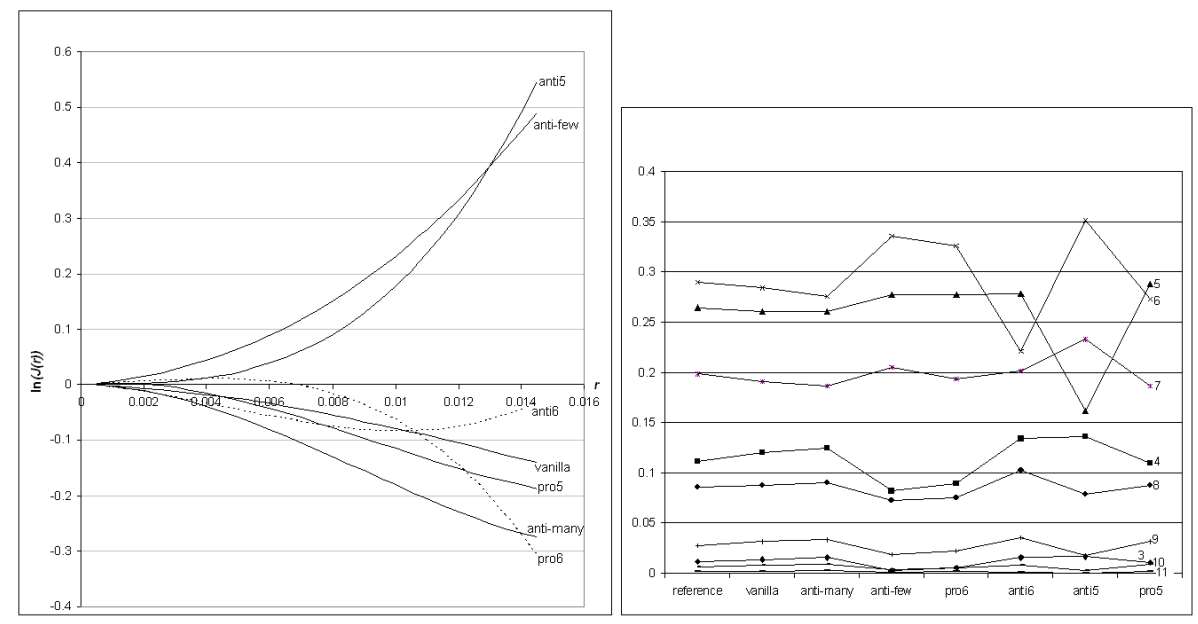

Figure 4: Comparison of $n$-processes $(N=2000$, after $T=12 N$ steps). Left: smoothed plots of $\ln \hat{J}(r)$. Right: Proportions of cells with given numbers of neighbours (given at the right-hand end). Standard error is approx. $1 \%$ of the given value in each case.

variable statistics and are not easily applied in practice. The statistic $R^{*}$ provides a crude but fairly sensitive measure of deviation from CSR. The NN EPMF is probably the most effective statistic for the $n$-processes, but it doesn't distinguish well between CSR and 'anti-many' models (and also between 'anti-few' and 'pro-6'), whereas the $J$-curves for these models are very different. The $J$-curves for these and for the 'sharp filters' on four and seven neighboured cells reveal the interesting fact that selection functions favouring cells with $m>6(m<6)$ Voronoi neighbours produced more (less, resp.) uniform [than CSR] configurations. The 'anti-few' and 'anti-5' processes produce very similar $J$-curves, but are distinguished by their effects on $R^{*}$ : 'anti-few' gives $R^{*}=0.107$, while 'anti-5' has $R^{*}=0.126$, which are significantly different from each other as well as being significantly below the CSR value of 0.135 . The low value for $R^{*}$ corresponds to less variablity in cell areas, so this is consistent with the upward $J$-curves. Notice also the unusual curvature of the 'anti-6' curve, a result which was confirmed by increasing the number of independent draws in this case to 75 . In all these cases, the $R^{*}$ and $\hat{J}(r)$ statistics gave consistent results, i.e. upward curves corresponded to $R^{*}<0.135$.

\subsection{Two-dimensional $v$-processes}

We study here the $v$-processes on $M=[0,1]^{2}$ with the scale-free selection functions $S_{v}(u)=u^{\alpha}$. Figure 5 shows a few examples of point patterns after a considerable period of evolution. There is a continuous gradation of patterns, from almost regularly spaced ones for $\alpha<0$ through increasing levels of clustering until we reach the threshold level at $\alpha=1$. For $\alpha>1$, the patterns become increasingly concentrated in a small region, usually in a corner of the square, as the number of steps increases.

For $\alpha \leq 1$ we can employ the same statistics as for the $n$-process. As in the onedimensional case, Thiel's redundancy measure $R^{*}$ serves well for inferring the value of $\alpha$, and also provides a good indicator of the stabilisation of the process, see Fig.6. From Figs.66, 3it appears that the two-dimensional processes settle into equilibrium at roughly the same rate as the one-dimensional processes. The $\ln \hat{J}(r)$ curves for $\alpha \in(-3,1)$ form a fan as expected, see Fig.7](left). More detailed analysis reveals surprising (statistically significant) changes in curvature that occur for $\alpha$ between -0.3 and -0.2 and between 0.5 and 0.6: it appears that a subtle phase change does indeed occur around these values. In 


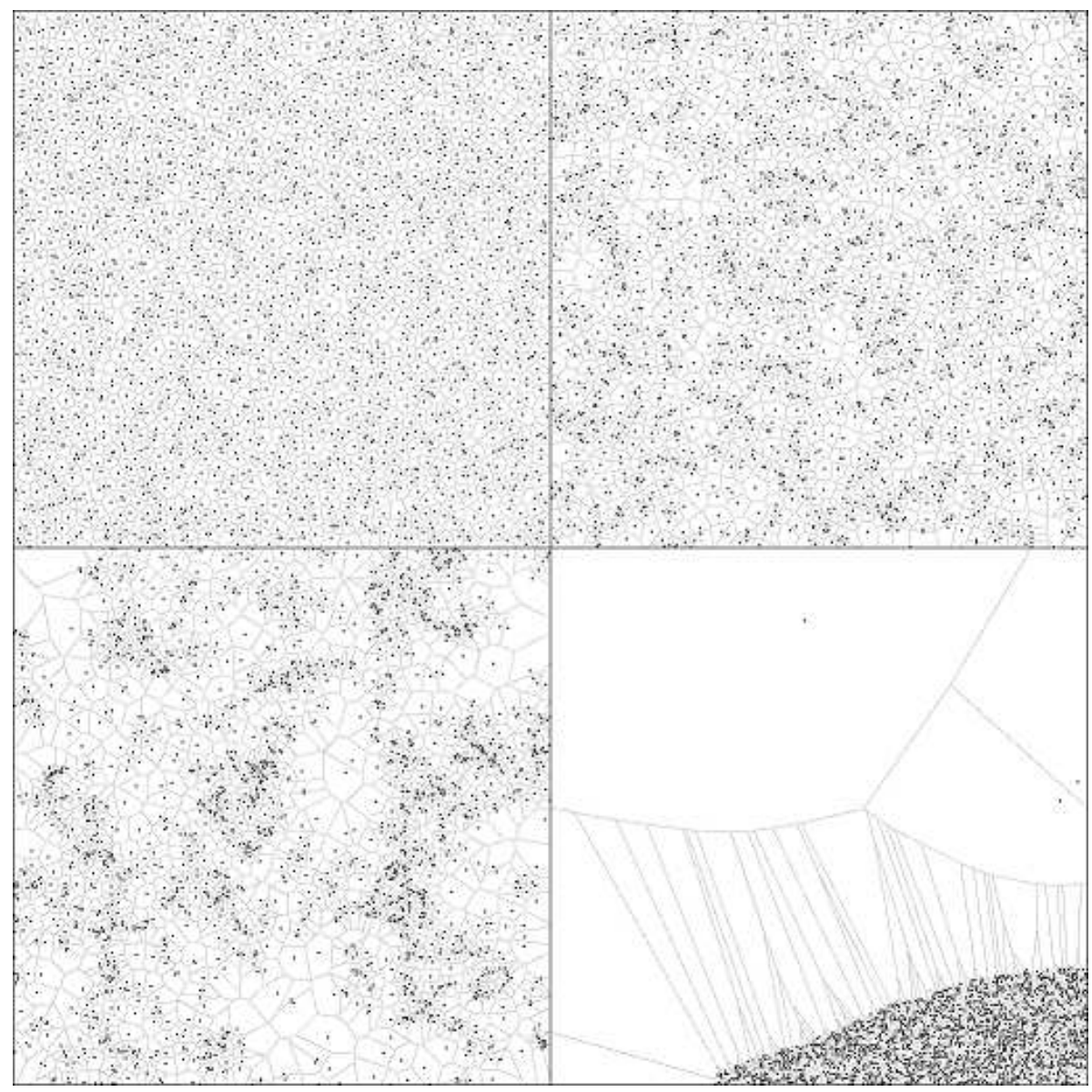

Figure 5: Realisations of the $v$-process with $S_{v}(u)=u^{\alpha}$ for some $\alpha$ values. Left-right, top-bottom: $\alpha=-3 ; 0.2 ; 1 ; 1.4$. In each case, $N=2000, T=12 N$.

Fig. [7(right) we have plotted the NN EPMF as a function of $\alpha$. Note that, as $\alpha$ increases (and so the amount of clustering), the proportions of cells with different numbers of edges tend to come together; in other words, the cell geometries become more variegated.
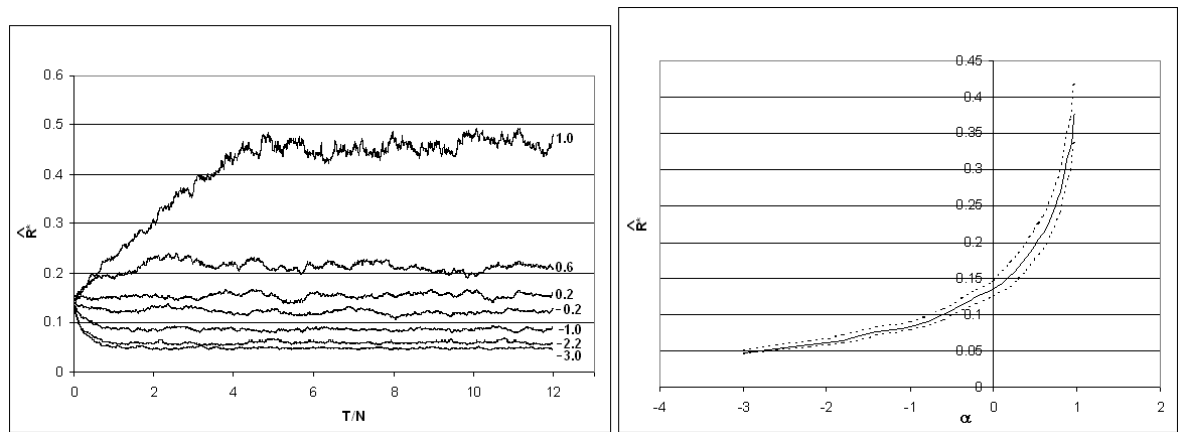

Figure 6: Thiel's redundancy measure for $v$-processes on $[0,1]^{2}\left(N=2000, S_{v}(u)=u^{\alpha}\right)$. Left: Evolution of $\left\{R^{*}\left(x_{T}\right)\right\}_{T \leq 12 N}$ for $\alpha=-3 ;-2.2 ;-1 ;-0.2 ; 0.2 ; 0.6 ; 1$. Right: $R^{*}$ vs $\alpha$ (after $T / N=12$ steps).

\section{Comparison with the area-interaction process}

The area-interaction point process (AIPP) of Baddeley and van Lieshout [3] is a model which successfully produces a range of different clusterings. Kendall [6] presented a prac- 


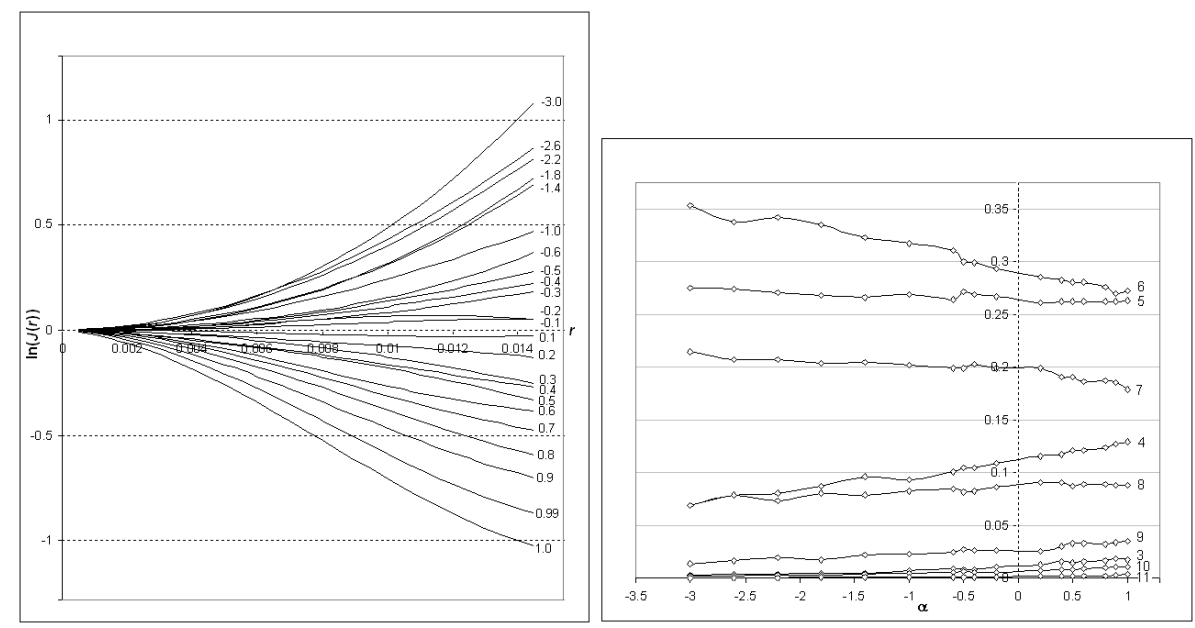

Figure 7: Graphical summary of results for $v$-processes on $[0,1]^{2}$ with $S_{v}(u)=u^{\alpha}$ for different $\alpha$ values $(N=2000, T=8 N)$. Left: Smoothed curves for $\ln \hat{J}(r)$ (numbers of independent draws range from $9(\alpha \leq-1.0)$ to $59(\alpha=0.5,0.6))$. Note the rapid change in the curve as $\alpha$ approaches 1 . Right: Proportions of cells with given numbers of neighbours (given at the right-hand end) as functions of $\alpha$.

tical method for 'perfect simulation' of this process. This is summarised in Ambler [1] from whose detailed account we derived our computer program for simulations.

Let $M=[0,1]^{2}$ and $\mathscr{M}$ be the space of all finite configurations of points in $M$. The AIPP is specified by the density of its distribution with respect to that of the unit rate Poisson process on $M$. Let $\lambda$ be Lebesgue measure on $M, G:=B(\rho)$ for a fixed $\rho>0$, and $\boldsymbol{x} \oplus G:=\{z: z=x+g, x \in \boldsymbol{x}, g \in G\}$ for $\boldsymbol{x} \in \mathscr{M}$. The above density is given by

$$
p(\boldsymbol{x})=C \beta^{\operatorname{card}(\boldsymbol{x})} \gamma^{-\lambda(\boldsymbol{x} \oplus G)},
$$

where $\beta, \gamma>0$ are parameters, $C$ a normalising constant. Note that the Poisson process on $M$ with constant rate $\beta$ has density $\beta^{\operatorname{card}(\boldsymbol{x})}$. The parameter $\gamma$ defines the interactive component of the process: when $0<\gamma<1$, configurations with high values of $\lambda(\boldsymbol{x} \oplus G)$ for a given $\operatorname{card}(\boldsymbol{x})$ are favoured, and so the interaction is described as repulsive, while $\gamma>1$ favours $\boldsymbol{x}$ with low $\lambda(\boldsymbol{x} \oplus G)$ and so constitutes the attractive case.

The expected number of points in $M$ is a complicated function of $\gamma, \beta$ and $\rho$. We chose $\rho=0.01, \gamma=\gamma_{1}^{10^{4}}$ with $\gamma_{1} \in[0.3,1.5]$, and adjusted $\beta$ so that the number of points produced in the simulations of the AIPP was close to 2000. This is because the AIPP doesn't re-scale in a simple way, so comparison with the Voronoi $v$-process, in particular in regard to the $J$-function, requires the samples to have roughly the same number of points.

Figures 8 [10 show the values of our three statistics for the AIPP. We observe a continuous range of degrees of clustering on either side of CSR (when $\ln J(r) \equiv 1$ ). It is interesting to compare these with those derived from the Voronoi $v$-process. In particular, we can match results which produce close values for $R^{*}$. We have done this for the cases of the AIPP with $\gamma_{1}=1.5$ and Voronoi $v$-process with $\alpha=0.5$ (for both processes, $R^{*} \approx 0.2$ ). The right-hand plots in Figs. [0 10] show that there is a significant difference in the $J$-function curves, but that the Voronoi NN EPMF's do not yield significant difference.

In summary, the $v$-processes and the AIPP's produce a range of clusterings depending on a continuous parameter. The difference in the resulting point patterns can be detected by the combination of our three statistics (a)-(c). 


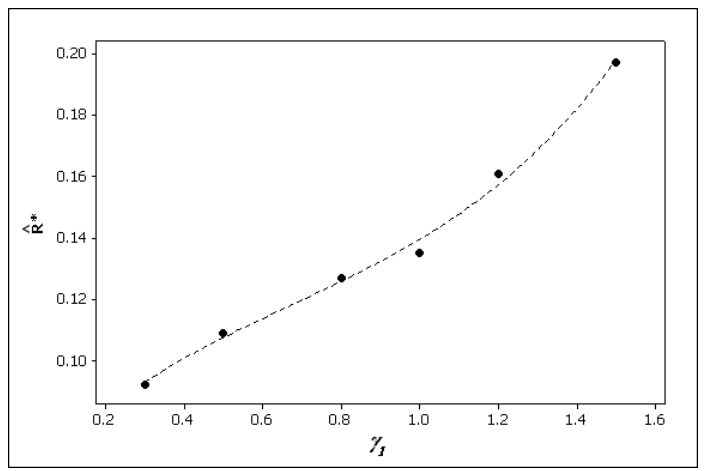

Figure 8: Plot of $R^{*}$ vs $\gamma_{1}$ for the AIPP as described above, with cubic regression line. Standard deviations of $R^{*}$ are close to 0.005 .
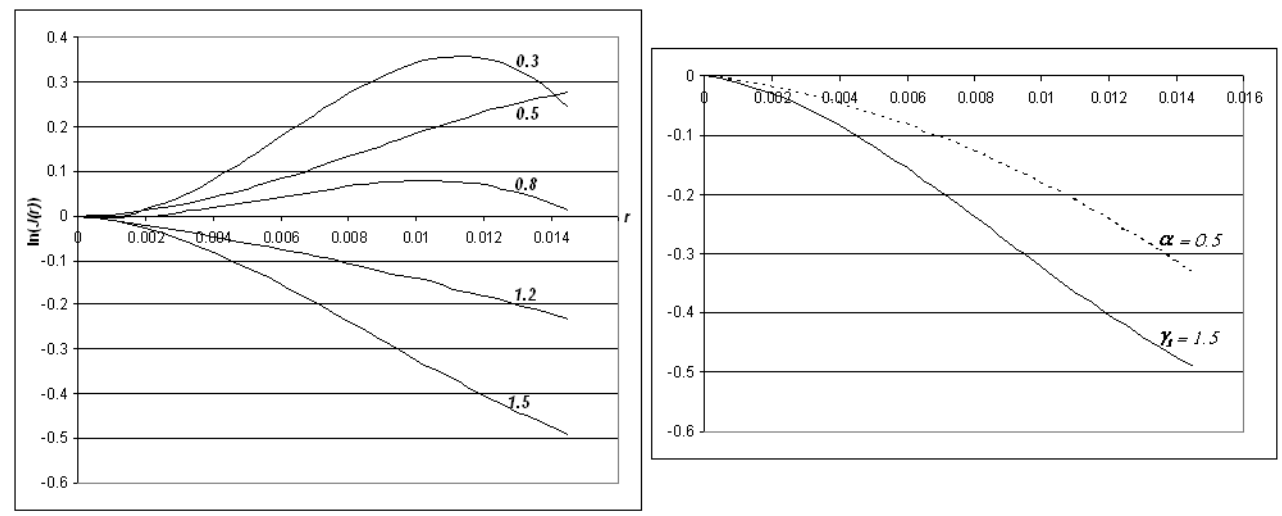

Figure 9: Left: Plots of smoothed $\ln \hat{J}(r)$ curves for the AIPP's with various values of $\gamma_{1}$, showing a range of attractive/repulsive effects. Right: The graph of $\ln \hat{J}(r)$ for the AIPP with $\gamma_{1}=1.5$ is compared to that for the scale-free Voronoi $v$-process with $\alpha=0.5$ (these two processes produce almost identical $R^{*}$ values and NN EPMF's).
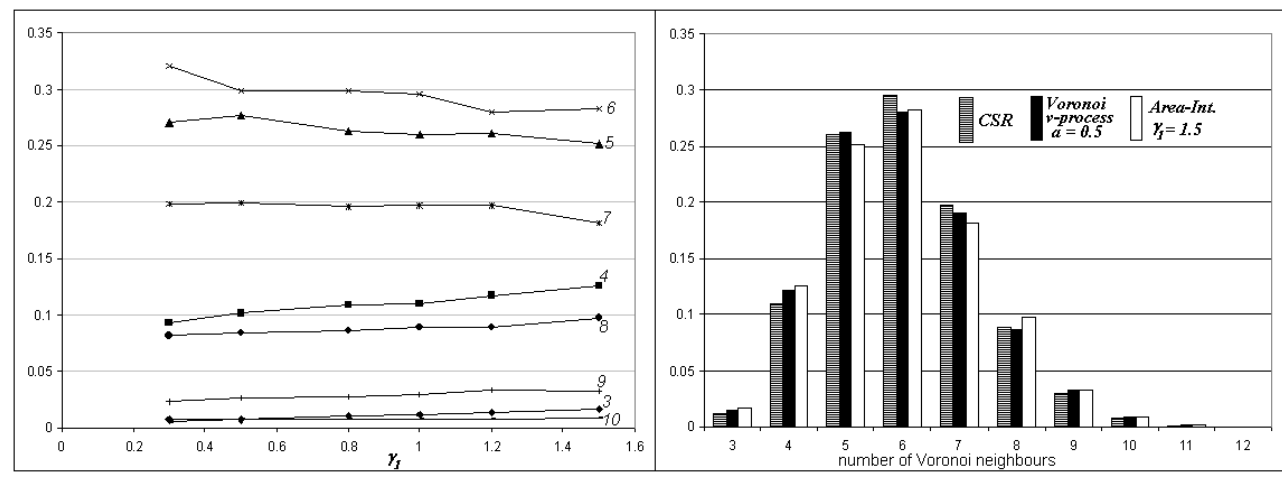

Figure 10: Left: Proportions of cells with given numbers of neighbours (given at the right-hand end) for the AIPP's, plotted vs $\gamma_{1}$. Right: Comparison of the NN EPMF's for the AIPP with $\gamma_{1}=1.5$, the Voronoi $v$-process with $\alpha=0.5$, and CSR. Standard errors are close to 0.005 .

\section{References}

[1] Ambler, G. K. (2002) Dominated Coupling From The Past and Some Extensions of the Area-Interaction Process. PhD thesis, Department of Mathematics, University of Bristol, U.K.

[2] Asmussen, S. (1987) Applied probability and queues. John Wiley, Chichester. 
[3] Baddeley, A. and van Lieshout, M. (1995) Area-interaction point processes. Annals of the Institute of Statistical Mathematics, 47, 601-619.

[4] Baddeley, A. And van Lieshout, M. (1996) A nonparametric measure of spatial interaction in point patterns. Statistica Neerlandica, 50, 344-361.

[5] Borovkov, K.A. And Odell, D. (2006) On spatial culling-immigration point processes based on Voronoi cells. (Submitted; avaliable as arXiv.org paper math.PR/0610606.)

[6] Kendall, W.S. (1998) Perfect simulation for the area-interaction point process. (1979) In: L. Accardi and C.C. Heyde (Eds.), Probability Towards 2000, 218-234, Springer, NY.

[7] Lenz, R. Redundancy as an Index of Change in Point Pattern Analysis. Geographical Analysis, 11, 374-388.

[8] Meyn, S.P. And Tweedie, R.L. (1992) Stability of Markovian processes I: Criteria for discrete-time chains. Adv. Appl. Prob. 24, 542-574.

[9] Okabe, A., Boots, B., Sugihara, K., And Chiu, S. (2000) Spatial Tessellations: Concepts and Applications of Voronoi Diagrams. John Wiley, Chichester. 